\title{
A 3D Human Liver Model of Nonalcoholic Steatohepatitis
}

\author{
Marion Duriez ${ }^{1}$, Agnes Jacquet ${ }^{1}$, Lucile Hoet ${ }^{1}$, Sandrine Roche ${ }^{1}$, Marie-Dominique Bock ${ }^{1}$, \\ Corinne Rocher ${ }^{1}$, Gilles Haussy ${ }^{1}$, Xavier Vigé ${ }^{1}$, Zsolt Bocskei ${ }^{1}$, Tamara Slavnic ${ }^{2}$, \\ Valérie Martin ${ }^{3}$, Jean-Claude Guillemot ${ }^{1}$, Michel Didier ${ }^{1}$, Aimo Kannt ${ }^{4,5}$, Cécile Orsini ${ }^{1}$, \\ Vincent Mikol ${ }^{1}$ and Anne-Céline Le Fèvre* ${ }^{1}$
}

${ }^{1}$ Translational Sciences, Sanofi, 1 Avenue Pierre Brossolette, Chilly-Mazarin, France; ${ }^{2}$ IT\&M STATS, Groupe IT\&M, Neuilly-surSeine, France; ${ }^{3}$ Non Clinical Biostatistics, Sanofi, Vitry sur Seine, France; ${ }^{4}$ Diabetes and Cardiovascular Research, Sanofi, Industriepark Höchst, Frankfurt, Germany; ${ }^{5}$ Present address: Institute of Experimental Pharmacology, Medical Faculty Mannheim, University of Heidelberg, Mannheim, Germany

\begin{abstract}
Background and Aims: To better understand nonalcoholic steatohepatitis (NASH) disease progression and to evaluate drug targets and compound activity, we undertook the development of an in vitro 3D model to mimic liver architecture and the NASH environment. Methods: We have developed an in vitro preclinical 3D NASH model by coculturing primary human hepatocytes, human stellate cells, liver endothelial cells and Kupffer cells embedded in a hydrogel of rat collagen on a 96-well plate. A NASH-like environment was induced by addition of medium containing free fatty acids and tumor necrosis factor- $\alpha$. This model was then characterized by biochemical, imaging and transcriptomics analyses. Results: We succeeded in defining suitable culture conditions to maintain the 3D coculture for up to 10 days in vitro, with the lowest level of steatosis and reproducible low level of inflammation and fibrosis. NASH disease was induced with a custom medium mimicking NASH features. The cell model exhibited the key NASH disease phenotypes of hepatocyte injury, steatosis, inflammation, and fibrosis. Hepatocyte injury was highlighted by a decrease of CYP3A4 expression and activity, without loss of viability up to day 10 . Moreover, the model was able to stimulate a stable inflammatory and early fibrotic environment, with expression and secretion of several cytokines. A global gene expression analysis confirmed the NASH induction. Conclusions: This is a new in vitro model of NASH disease consisting of four human primary cell-types that exhibits most features of the disease. The 10-day cell viability and cost effectiveness of the model make it suitable for medium throughput drug screening and provide attractive avenues to better understand disease physiology and to identify and characterize new drug targets.
\end{abstract}

Citation of this article: Duriez $M$, Jacquet $A$, Hoet $L$, Roche $S$, Bock MD, Rocher C, et al. A 3D human liver model of nonalcoholic steatohepatitis. J Clin Transl Hepatol 2020;8(4): 359-370. doi: 10.14218/JCTH.2020.00015.

Keywords: 3D liver model; Human primary cells; Key features of NASH. Abbreviations: ECM, extracellular matrix; HSC, hepatic stellate cell; KC, Kupffer cell; LEC, sinusoidal endothelial cell; NAFLD, nonalcoholic fatty liver disease; NASH, nonalcoholic steatohepatitis; $\mathrm{PHH}$, primary human hepatocytes. Received: 2 March 2020; Revised: 19 March 2020; Accepted: 1 June 2020

*Correspondence to: Anne-Céline Le Fèvre, Translational Sciences, Sanofi, 1 Avenue Pierre Brossolette, 91385 Chilly-Mazarin, France. Tel: +33-160495815, E-mail: anne-celine.le-fevre@sanofi.com

\section{Introduction}

Nonalcoholic fatty liver disease (NAFLD) is an umbrella term that comprises a large spectrum of liver injuries, varying in severity but all leading to fibrosis. Among these, nonalcoholic fatty liver (NAFL) refers to hepatic steatosis alone, which is very common ${ }^{1}$ and driven by the accumulation of intracellular lipid droplets. Furthermore, nonalcoholic steatohepatitis (NASH) is defined as a more serious pathogenesis, having inflammatory foci, hepatocyte damage, and fibrosis. Adverse hepatic outcomes related to NASH may include cirrhosis, liver failure, and hepatocellular carcinoma. ${ }^{2}$ NAFLD is associated with obesity and features of metabolic syndrome, including hypertension, dyslipidemia, central adiposity, insulin resistance, or diabetes. ${ }^{3,4}$ Moreover, NASH with advanced fibrosis has been linked to increased overall and liver-related mortality. ${ }^{5}$

Today, bariatric surgery is the most efficient procedure to reverse NASH and fibrosis in obese patients. ${ }^{6}$ Weight loss induced by diet and exercise has also been shown to be effective in resolving NASH and improving hepatic fibrosis. ${ }^{7}$ Despite a marked increase in prevalence, though NASH is still an orphan disease, with no approved drugs for its treatment. Thus, a better management approach for NAFLD and the development of new drugs and therapeutic options are urgently needed. ${ }^{8}$ Although there has been steady progress in understanding NASH pathogenesis, the identification of therapeutic targets and the advancement of drug development have shown limited progress, mainly due to the lack of predictive preclinical models. Several animal models have been developed to study NAFL and NASH, but they do not accurately depict the human pathology, presumably because of NAFL/NASH heterogeneity. ${ }^{9}$

In drug discovery, hepatic in vitro models have been used to assess drug clearance and hepatotoxicity by investigating metabolism, enzyme induction, and transporter function. Monolayer cultures of isolated primary rat or human hepatocytes remain the main investigative tools for drug testing. These 2D models have shown several limitations, including a short lifetime and loss of function, likely resulting from dedifferentiation of primary hepatocytes $(\mathrm{PHH}) .{ }^{10}$ Precisioncut liver slices, which contain $\mathrm{PHH}$ as well as liver nonparenchymal cells have also shown reduced lifetime; thus, impairing the development of liver chronic disease models and robust drug testing. 
More recently, 3D cultures have been developed to improve cell survival and to provide a more natural tissue-like environment. In the liver, the extracellular matrix (ECM) behaves as a scaffold for surrounding cells, and in vitro artificial matrix aims to support ECM functions by promoting cell adhesion, cell differentiation, and cell-to-cell communication. ${ }^{11-13}$ 3D cultures of hepatic cell lines or human $\mathrm{PHH}$ embedded in artificial scaffolds were shown to modify gene and cell surface receptor expression toward more mature-like phenotypes, resulting in the maintenance of hepatocyte polarization and functionality. Furthermore, it has been shown that collagen gels enhanced mechanical properties with good cell adhesion and a high survival rate for hepatocytes. ${ }^{10}$ Thus, the 3D cell culture systems appear to be more representative of hepatocyte physiology in liver tissue and provide opportunities to develop extended in vitro models of NASH and NAFLD.

The development of in vitro human 3D models to mimic liver architecture has been undertaken by several groups, especially for drug safety assessment. ${ }^{14}$ The process has included layered cocultures and cocultures on micropatterned surfaces, spheroids and bioprinted liver tissue. ${ }^{14}$ Many of these models have been grown within specialized microfluidic devices, to provide nutrients and oxygen transport. ${ }^{14}$ Few 3D coculture studies have been designed as NAFL or NASH disease models to display key pathogenic phenotypes. Thus, steatosis has been observed in NAFLD models supplemented with high concentrations of oleic and palmitic acids. Increased lipid accumulation was found to be associated with altered gene expression and activity of several CYP450 enzymes. Only limited cytokine release was reported, likely due to the absence of Kupffer cells (KCs) in these in vitro systems. Therefore, such 3D models highlighted the need to coculture additional cell types that could further incorporate features of inflammation and fibrosis and better reflect disease progression.

Feaver et al. ${ }^{15}$ set up a 3D model with $\mathrm{PHH}$, monocytederived macrophages and hepatic stellate cells (HSCs) in hemodynamic and transport conditions. Correlations between the in vitro model and human biopsies were evidenced by transcriptomics, lipidomics, and functional analysis. This model requires differentiating monocytes into macrophages, which is time consuming, and yields only M2 macrophages, a nonphysiological macrophage subpopulation. In addition, this hemodynamic system is unsuitable for high-throughput screening.

In this paper, we report on a new 3D coculture model combining $\mathrm{PHH}$ with HSCs, sinusoidal endothelial cells (LECs) and KC. Activated HSCs play a principal role in fibrosis initiation and development through the production of collagen, while $\mathrm{KCs}$ are involved in liver damage and inflammatory processes. LECs were more recently shown to play a pivotal role in NAFL/ NASH progression. ${ }^{16}$ This model was characterized biochemically and transcriptionally, and displayed some key features of hepatic injury, steatosis, inflammation, and early fibrosis. Its 10-day cell viability, as well as its reasonable cost-effectiveness, make it compatible for medium throughput screening in 96-well plates. This coculture model thus provides a valuable platform to better understand NASH disease progression, and to evaluate drug targets and compound activity.

\section{Methods}

The liver microenvironment under NAFLD and NASH disease conditions includes many circulating risk factors, which were incorporated into cell media to promote in vitro NAFLD or
NASH-relevant phenotypes. Those risk factors include high glucose and insulin concentrations, excess of free fatty acids and endotoxins. For steatosis induction, free fatty acids (palmitic and oleic acids) were used, which lead to the accumulation of intracellular lipid droplets. This lipotoxic phenotype is the most commonly used stimuli for NAFLD in vitro models, as it can also lead to an increase of inflammatory cytokines levels. ${ }^{17,18}$ Tumor necrosis factor- $\alpha$ was added to induce the inflammation process and activate the KCs. Cells were embedded in a hydrogel of rat collagen in 96-well plates and the NASH environment was induced by adding a media containing free fatty acids and tumor necrosis factor- $\alpha$. Considering the main characteristics of NASH pathology, hepatocyte injury, steatosis, inflammation and fibrosis were assessed biochemically and via transcriptomics.

\section{Cell 3D coculture in hydrogels/matrix of collagen}

PHH were obtained from Lonza (Walkersville, MD, USA), and HSCs, KCs and LECs were provided by Samsara (San Diego, CA, USA). PHH, HSCs and LECs were seeded in red phenolfree William E medium (A12176; Gibco, Invitrogen Corp., Waltham, MA, USA) supplemented with $5 \%$ fetal bovine serum, primary hepatocyte thawing and plating supplements solution (CM3000; ThermoFisher Scientific, Waltham, MA, USA) and $1 \%$ of a non-essential amino acids solution (11140050; Gibco).

PHHs, LECs and hepatic stellate primary cells were embedded at $0.5 \times 10^{6}, 0.1 \times 10^{6}$ and $0.1 \times 10^{6}$ cells $/ \mathrm{mL}$, respectively, in a half RAFT ${ }^{\mathrm{TM}}$ 3D collagen hydrogel (016-0R92; Lonza) in 96-well plates, as recommended by the provider. Cells were cocultured in DMEM (low-glucose, pyruvate, no glutamine, no red phenol; 11054; Gibco) supplemented with bovine serum/ free fatty acid-free $0.125 \%$ solution (A7030; Sigma-Aldrich, St Louis, MO, USA), $50 \mathrm{U} / \mathrm{mL}$ penicillin/streptomycin (15140122; Gibco), dexamethasone $0.1 \mu \mathrm{M}$, ITS-G $1 \mathrm{X}$ (41400045; Gibco), GlutaMAX ${ }^{\mathrm{TM}}$ 1X (35050061; Gibco), HEPES $15 \mathrm{mM}$ (15630080; Gibco), non-essential amino acids solution 1X (11140050; Gibco), acid L-ascorbic $2.5 \mathrm{mg} / \mathrm{mL}$ (A4403; Sigma-Aldrich) and glucagon $0.1 \mu \mathrm{g} / \mathrm{mL}$ (G2044; Sigma-Aldrich). This liver coculture medium is named "healthy media". After 3 days of culture, the cocultures were incubated either in healthy media or in a media mimicking the NASH environment and supplemented with glucose 25 mM (Sigma-Aldrich), oleate acid $40 \mu \mathrm{M}$ (SigmaAldrich), palmitate acid at $60 \mu \mathrm{M}$ (Cayman Chemical, Ann Arbor, MI, USA) and tumor necrosis factor- $\alpha 5 \mathrm{ng} / \mathrm{mL}$ (PeproTech, Cranbury, NJ, USA). Healthy and NASH medium were changed every 2-3 days. At day $6, \mathrm{KCs}$ were added to the coculture at $0.2 \times 10^{6}$ cells $/ \mathrm{mL}$. Supernatants and embedded cells were sampled on days $3,6,8,10,13$ and 15 for analysis.

\section{Viability and hepatocyte metabolism}

Viability was assessed by measurement of adenosine triphosphate using the CellTiter-Glo 3D Assay (G9681; Promega, Madison, WI, USA). PHH metabolism was measured through CYP3A4 activity with the Luciferin-IPA CYP3A4-P450 Glo Assay (V9002; Promega).

\section{D primary liver cell coculture immunostaining}

3D hydrogels were fixed with 4\% Paraformaldehyde/phosphate-buffered saline for $15 \mathrm{~min}$ at room temperature and 
immunostainings were performed. $\mathrm{PHH}$ cell membrane staining was performed with a rabbit anti-cytokeratin 18 antibody (EPR17347, \#Ab181597; Abcam, Cambridge, UK) and an Alexa Fluor 594 conjugated goat anti-rabbit IgG secondary antibody (\#A11012; Molecular Probes, Eugene, OR, USA). HSCs were stained with a mouse anti- $\alpha$-smooth muscle actin antibody (\#A5228; Sigma-Aldrich) and an Alexa Fluor 680 conjugated goat anti-mouse IgG secondary antibody (\#A21057; Molecular Probes). KCs were stained with an FITC conjugated anti-CD68 antibody was used (KP1, \#FCMAB205F; Sigma-Aldrich), nuclei were labeled using NucBlue live (\#R37610; ThermoFisher Scientific). Image acquisitions were performed with a Leica SP8X confocal microscope, using $\times 40$ water objective, Z-stack of $Z=30 \mu \mathrm{m}$ zoom 0.8. 3D reconstitution was performed with IMARIS software 9.1.2.

\section{Triglyceride content}

Triglycerides content was measured on coculture supernatants with the PicoProbe Triglyceride Quantification Assay (K614-100; BioVision Inc., Milpitas, CA, USA). Intrahepatic lipid droplets were stained with the LipidTox Green probe (H34475; ThermoFisher Scientific) and PHH cell membrane with CK18 immunostaining. Images of 3D cocultures were acquired with a Leica SP8X confocal microscope, using $\times 40$ water immersion objective and a Z-stack of $Z=30 \mu \mathrm{m}$, zoom 0.8 . 3D reconstitution was performed with IMARIS software 9.1.2.

\section{Cytokine/metalloprotease release}

The cytokine content in coculture supernatants were measured using the U-plex Biomarker Human Group 1 Kit (K15067L-2; MSD Technology, Rockville, MD, USA). Samples were diluted at $1 / 4$ for IL6, CXCL8 and CCL2 measurement, and no dilution was performed for CXCL10 quantification. The concentration of secreted metalloproteinase MMP2 was quantified with the Human MMP2 Ultrasensitive Kit (K151FYC-2; MSD Technology) with a $1 \frac{1}{2}$ dilution.

\section{Statistical methodology of biochemical parameters}

To compare NASH and healthy models, a two-way ANOVA was performed on each parameter, with group treatment (healthy or NASH), day and their interaction as fixed-effects factors, and with experiment and interaction of experiment, group and day as random effect factors. Comparisons of NASH versus healthy were provided for each day and no correction for multiplicity was done. To analyze the kinetics of the healthy model, a one-way ANOVA was performed on each parameter, with day as the fixed-effect factor and experiment and experiment by day as random effect factors. A Bonferroni-Holm's correction was applied on $p$-values to compare the following: day 6 vs. day 3 , day 8 vs. day 6 , day 10 vs. day 8 for comparison of both conditions; and day 10 vs. day 13 and day 13 vs. day 15 for healthy time course assessment. Either a log or rank transformation was applied on studied parameters, and fold-changes were calculated. For log-transformed variables, the differences estimated from the model and their confidence intervals were back-transformed by using an exponential function. For rank-transformed variables, fold-change and confidence intervals were estimated by Hodges-Lehmann's method.

\section{RNA extractions}

Hydrogels embedded cocultures containing $0.2 \times 10^{6}$ cells per well, were rinsed twice with cold phosphate-buffered saline and stored at $-80^{\circ} \mathrm{C}$ until use. For RNA extraction, two wells were lysed in a final volume of $700 \mu \mathrm{L}$ of Qiazol lysis reagent (Qiagen, Hilden, Germany). Lysates were homogenized using CK14-2 mL tubes in a Precellys tissue homogenizer instrument (Bertin Instruments, Montigny-le-Bretonneux, France). The lysates were collected and $140 \mu \mathrm{L}$ of chloroform were added, vortexed and centrifuged at $12000 \mathrm{rpm}$ for 15 minutes at $4^{\circ} \mathrm{C}$. Recovered aqueous phase was processed with an on-column DNAse treatment and a RNeasy mini-kit (Qiagen) as recommended by the provider. RNAs were recovered in $30 \mu \mathrm{L}$ of RNase-free water. RNA quality was determined by RNA LabChip with a 2100 Bioanalyzer (Agilent Scientific Instruments, Santa Clara, CA, USA). Samples with RNA integrity number $>7.4$ were further processed and their concentration was quantified by Xpose spectrophotometer (Trinean, Unchained labs, France).

\section{RNA libraries and sequencing}

RNA-Seq libraries were generated with $15 \mathrm{ng}$ of total RNA. cDNAs were generated with the SuperScript VILO cDNA Synthesis Kit (ThermoFisher Scientific) and the following steps for library construction were performed using the "AmpliSeq for Illumina Transcriptome Human Gene Expression Panel" according the accompanying reference guide (Illumina, San Diego, CA, USA).

Libraries were quantified and qualified, respectively, by Qubit (Invitrogen) and Bioanalyzer (Agilent). Libraries were pooled (equimolar concentration at $4 \mathrm{nM}$ ), denatured and diluted to a final concentration of $1.4 \mathrm{pM}$. Sequencing was performed on the Illumina NextSeq500 with NextSeq 500/ 550 High Output v 2 Kit and sequencing parameters of $2 \times 151$ base pair pair-end, dual index ( $2 \times 8$ base pairs).

Generated raw files were converted into FASTQ files and analyzed on Array Studio (V10.0.1.81; Omicsoft, Qiagen). Briefly, raw data QC was performed, then a filtering step was applied to remove reads corresponding to rRNAs as well as reads having low quality score. Mapping and quantification were performed using OSA4 [1C] (Omicsoft Sequence Aligner, version 4). ${ }^{19}$ Reference Human.B38 genome was used for mapping and genes were quantified based on RefSeq gene annotations. Differentially-expressed transcripts were identified with DESeq2. ${ }^{20}$ The variable multiplicity being taken into account and false discovery rate adjusted $p$-values calculated with the Benjamini-Hochberg correction. ${ }^{21}$ Significant differentially-expressed genes were defined as $p<0.05$ after adjustment for false discovery and average fold-change between condition replicates of $>1.8$. The differentiallyexpressed genes were further analyzed using Ingenuity Pathway Analysis (Qiagen; https://www.qiagenbioinformatics.com/products/ingenuity-pathway-analysis). ${ }^{22}$

\section{Results}

\section{Human liver primary cells 3D coculture characteristics in healthy conditions}

Setting up a human in vitro NASH disease model requires developing a coculture containing the different cells involved in the pathogenesis and maintaining it over an extended 
period of time in culture to induce this chronic disease. We developed a 3D coculture with primary cells using the RAFT ${ }^{\mathrm{TM}}$ biological rat collagen hydrogel embedding $\mathrm{PHH}$, HSCs, KCs and LECs. The addition of LECs as feeder cells in the 3D liver coculture allowed us to stabilize the model by improving cell viability from 1 week up to 2 weeks (data not shown). $\mathrm{PHH}$, HSCs and LECs were seeded in the hydrogel of collagen at a $5: 1: 1$ ratio, respectively, reproducing the human liver ratio, and cultured in a 96-well plate in a homemade cell culture media named 'healthy media' (Fig. 1A). At day 6, KCs were added to the coculture since they did not tolerate the embedding process (data not shown) but were able to enter the hydrogel once formed. Several media, where Obeticholic acid/Palmitic acid concentrations, glucose concentration and tumor necrosis factor were varied, were tested to maintain viability of the four cell types. Only the homemade liver coculture optimized media, described in the Materials and Methods section, allowed us to obtain a viable coculture of the four cell types up to day 10 (Fig. 1B).

To assess the suitability of the model, coculture viability, cytokine secretion and lipid droplet content were measured from day 3 to day 15 in four independent experiments. The human 3D liver coculture at a basal level displayed a good viability from day 3 to day 15 , as reflected by adenosine triphosphate level (Fig. 2A). PHH CYP3A4 enzymatic function was evaluated and it remains stable from day 3 up to day 13 (Fig. 2B). Inflammatory cytokines and chemokines IL6, CXCL8 and CXCL10 were measured in coculture supernatants. Basal levels of secreted IL6 and CXCL8 remained stable from day 3 to day 15 , with a nonsignificant increase at day 8 resulting from the addition of KCs at day 6 (Fig. $2 \mathrm{C}$ and 2D). CXCL10 was almost undetectable before day 8 , after $\mathrm{KCs}$ addition, and its secretion in cell supernatants remained stable from day 8 to day 15 (Fig. 2E). The triglyceride content of the human liver 3D coculture was quantified, and PHH lipid droplets were observed by immunofluorescence via Green LipiTox and a cytokeratin-18 containing (Fig. 2F and 2G, respectively). A high basal level of triglycerides with no significant variation was detected from day 3 to day 15 (Fig. 2F). This result was correlated with the observation of an important intracellular lipid droplet staining in $\mathrm{PHH}$ from day 3 to day 10 (Fig. 2G). Of note is that the PHH triglyceride content in human liver 3D coculture was higher than expected. Several media were tested on $\mathrm{PHH}$, including William's $\mathrm{E}$ media. All of them induced an elevated intracellular lipid droplet content (Supplementary Fig. 1) for unknown reasons and the cell coculture showed a lower viability than the homemade media.

Together, these results support a long-term viability and the stable expression of NASH-induced key features that can be directly quantified in the human 3D liver primary coculture under healthy conditions up to 15 days (Supplementary Table 1).

A transcriptomic analysis of human 3D liver primary coculture under the healthy condition was performed. Gene expression analysis was only performed on samples from days 3,8 and 10 ( $n=3 /$ condition) as the total RNA quantity extracted at day 13 and day 15 was too low for gene expression studies.

The expression of genes related to $\mathrm{PHH}$ activities, including the efflux transporters $A B C C 2, A B C B 1$ and $A B C B 11$, was first investigated. These genes are consistently detected during the time course of the coculture until day 10 (Supplementary Table 2) as well as UGT1A1, a gene encoding the phase II UDP-glucuronosyltransferase enzyme, with a foldchange $<2$ and/or with a $p$-value $>0.05$. Furthermore, CYP7A1 cytochrome P450 gene was expressed from day 3 to day 10 , with no significant difference (Supplementary Table 2), CYP3A4 expression, which was decreased at day 8 and day 10 compared to day 3, was stable from day 8 to day 10 (Supplementary Table 2) and was previously observed to be functional from day 3 to day 13 in 3D coculture (Fig. 2B). Together, these genes reflect the polarization, functionality and metabolic activity of mature $\mathrm{PHH}$ in human 3D liver primary coculture up to 10 days in vitro.

As expected, the expression of IL6, CXCL8 and CXCL10 inflammatory cytokine genes is observed. IL6 expression was stable between day 3 and day 10 (Supplementary Table 2), whereas CXCL8 and CXCL10 were significantly down- and upregulated from day 3 to day 8 , resulting from the addition of $\mathrm{KCs}$ but which remained stable from day 8 to day 10 (Supplementary Table 2). IL6, CXCL8 and CXCL10 gene expression results are in line with their secretion observed in 3D coculture supernatants (Fig2. C, D and E). The expression of
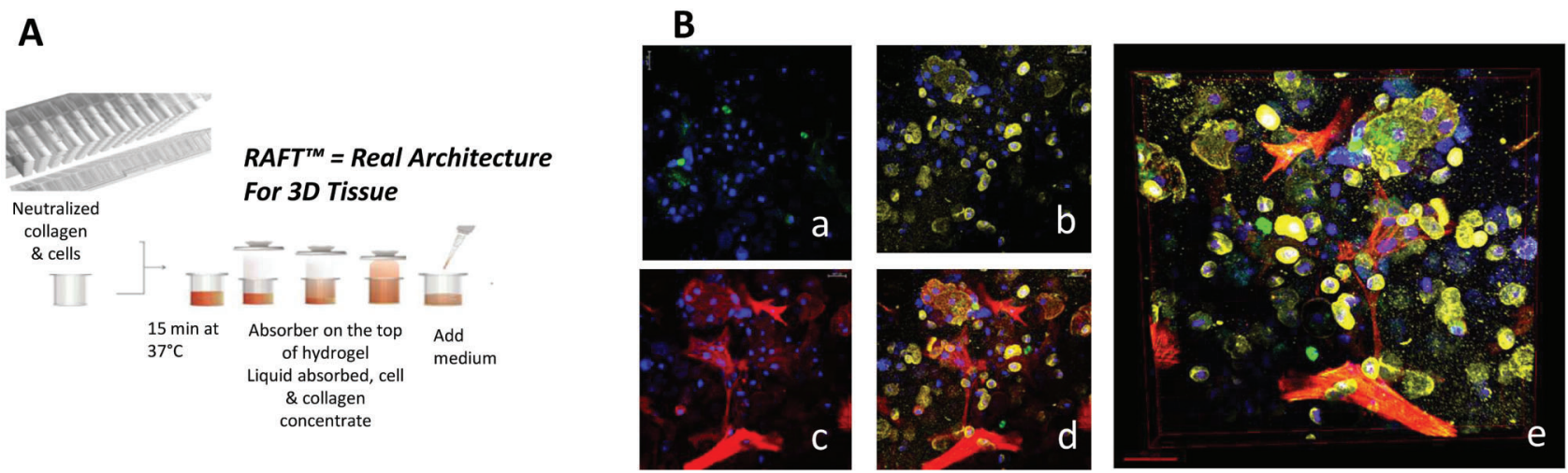

Fig. 1. Set-up of human liver primary coculture in a 3D environment.

(A) Real architecture for 3D model with RAFT ${ }^{\mathrm{TM}}$ system methodology illustration. (B) PHH-KC-HSC-LEC coculture at 10 days in 3D collagen matrix with healthy media. (a) KC staining with CD68 in green and nucleus-labeled in blue. (b) PHH staining with CK18 in green and nucleus labeled in blue. (c) HSC staining with $\alpha$-smooth muscle actin in red and nucleus labeled in blue. (d) Merge. (e) 3D reconstitution was performed with IMARIS software 9.1.2. 

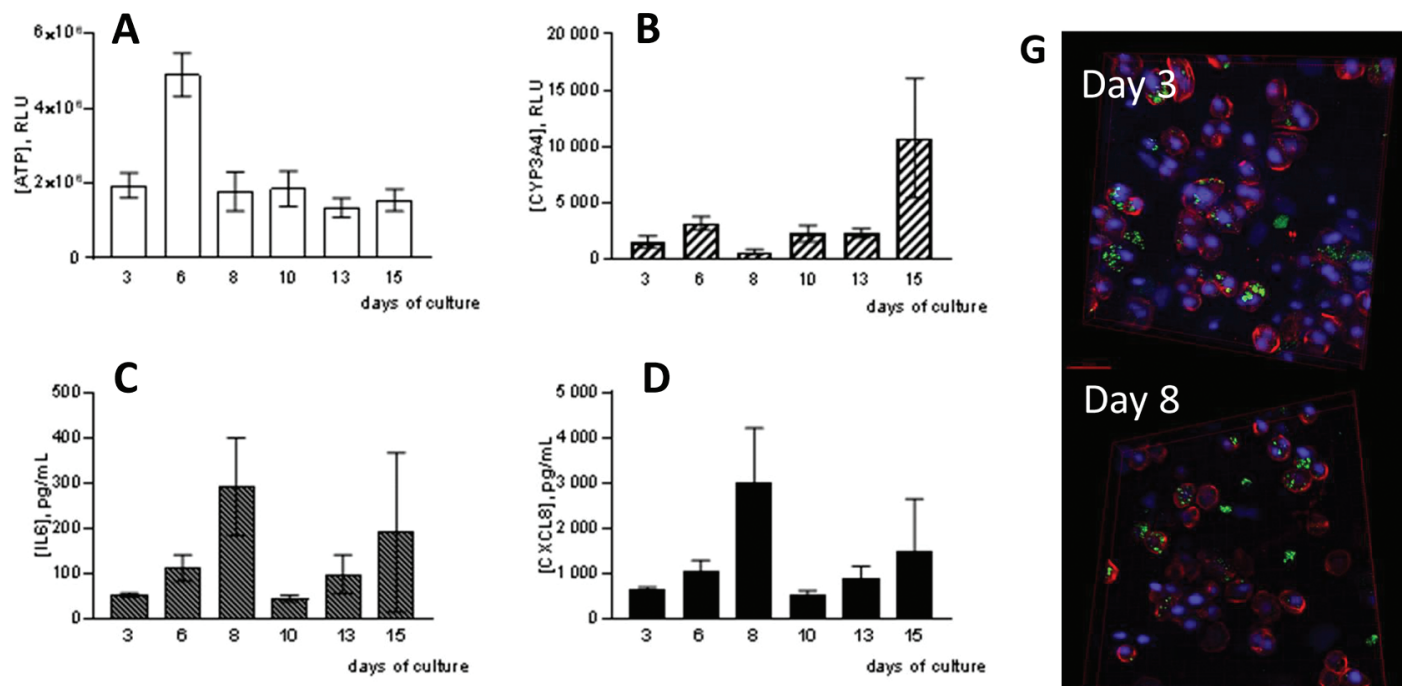

$\mathbf{E}$

$\mathbf{F}$
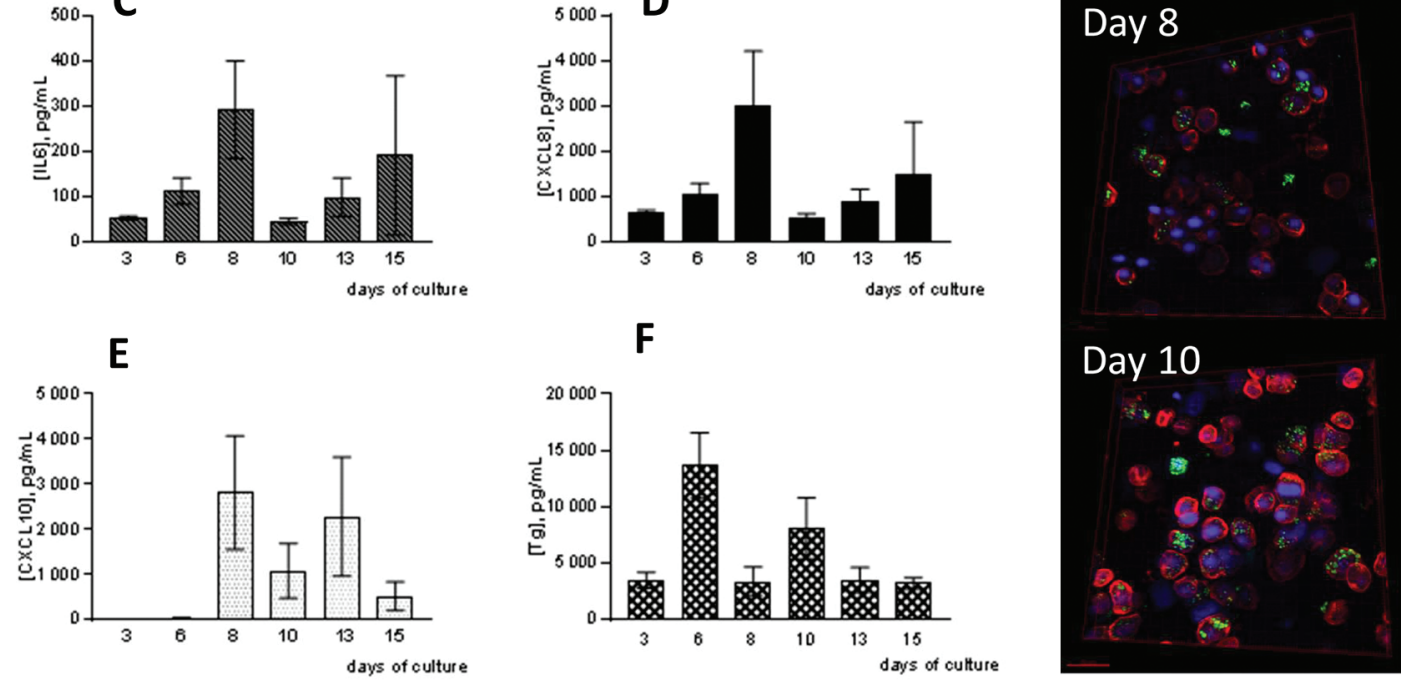

Fig. 2. Human 3D liver primary coculture characterization.

(A) Adenosine triphosphate intracellular in RLU. (B) CYP3A4 activity in RLU. (C) IL6 (D) CXCL8 and (E) CXCL10 in pg/mL. (F) Triglyceride content in $\mathrm{pg} / \mathrm{mL}$. (G) Kinetics of lipid droplet content in liver coculture in 3D collagen matrix, healthy condition. PHH staining of CK18 in red, nuclear in blue and lipid droplet in green. ${ }^{*} p<0.050,{ }^{*} p<0.010, * * * p<0.001$, two-way ANOVA with random effect followed by Bonferroni-Holm correction

Col1A1 gene, encoding collagen compounds remained stable from day 3 to day 10 (Supplementary Table 2), whereas ACTA2 gene, encoding the $\alpha$-smooth muscle actin protein, slightly increased with a 2.8 -fold-change and 2.4 -foldchange at day 8 and day 10 , as compared to day 3 , but which remained stable from day 8 to day 10 (Supplementary Table 2). The ACTA2 gene increased from day 3 to day 8 , probably resulting from the addition of KCs since the latter have been shown previously to activate HSCs through soluble factors. ${ }^{23}$ Together, these results showed that HSCs are not activated by a bystander effect of the coculture under healthy conditions.

We succeeded in defining suitable culture conditions, including medium composition and chronological cell addition, to maintain the four liver primary cell types in 3D coculture up to 10 days in vitro. Furthermore, these culture conditions gave the lowest level of steatosis, together with reliable low levels of inflammation and fibrosis.

\section{Human 3D liver NASH model display PHH injury and steatosis}

The human in vitro 3D NASH model was set up by culturing the human primary 3D liver coculture with a custom medium mimicking a $\mathrm{NASH}$-like disease environment added from day 3. This medium contained free oleic and palmitic fatty acids at
$100 \mu \mathrm{M}$ with a 2:3 ratio, as well as tumor necrosis factor- $\alpha$ at $5 \mathrm{ng} / \mathrm{mL}$.

The adenosine triphosphate levels measured in the human $3 \mathrm{D}$ liver coculture did not show a significant difference between the healthy and NASH culture conditions and indicated a good viability which was maintained until day 10 (Fig. 3A). ABCC2 and $A B C B 11$ gene expressions were not modulated during the coculture, neither in the NASH nor in the healthy models, showing a stable $\mathrm{PHH}$ mature phenotype up to 10 days in vitro (Fig. 3B and Supplementary Table 3). Cytochrome P450 CYP3A4, the expression of which is known to be reduced in $\mathrm{NASH}$, displayed a reduced activity and gene expression in the NASH model, as compared to healthy coculture but without reaching statistical significance (Fig. 3C). However, at day 10, three out the four experiments showed a decreased CYP3A4 activity (Fig. 3C), which correlated to a reduced gene expression at day 10 (Fig. 3D and Supplementary Table 3).

$\mathrm{PHH}$ steatosis assessed by triglyceride quantification in coculture did not show significant differences between healthy and NASH culture conditions (Fig. 4A). Intracellular lipid droplet staining is observed in both conditions but without noticeable differences at day 8 and day 10 (Fig. 4B). As mentioned before, $\mathrm{PHH}$ display a high basal level of triglycerides. This feature was observed for all the media tested, supporting the finding of PHH viability in the $3 \mathrm{D}$ collagen matrix and being consistent with biochemical quantification of steatosis. 


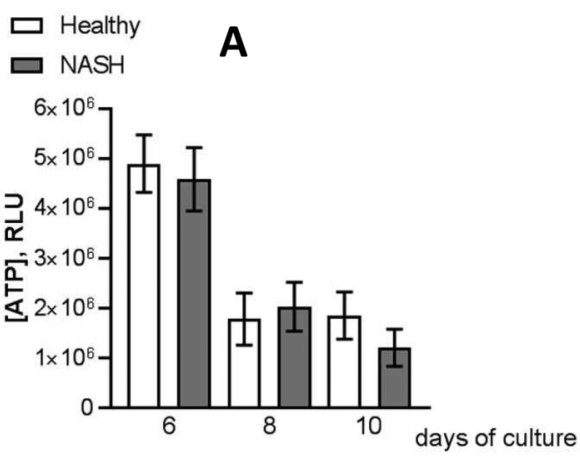

Day 10 of culture

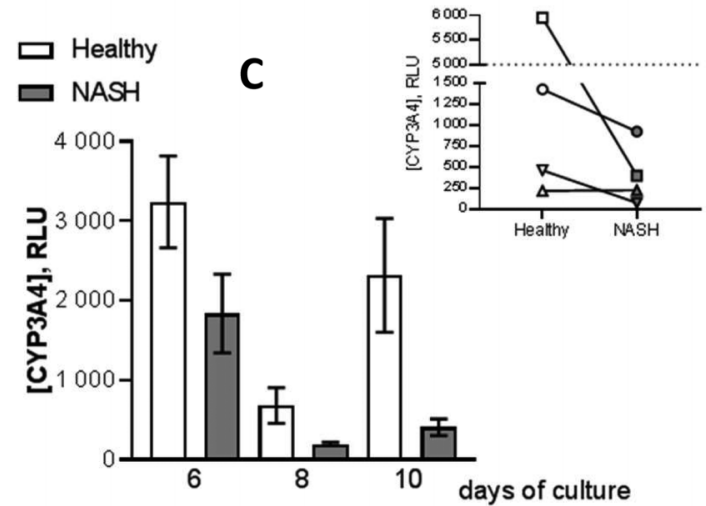

B
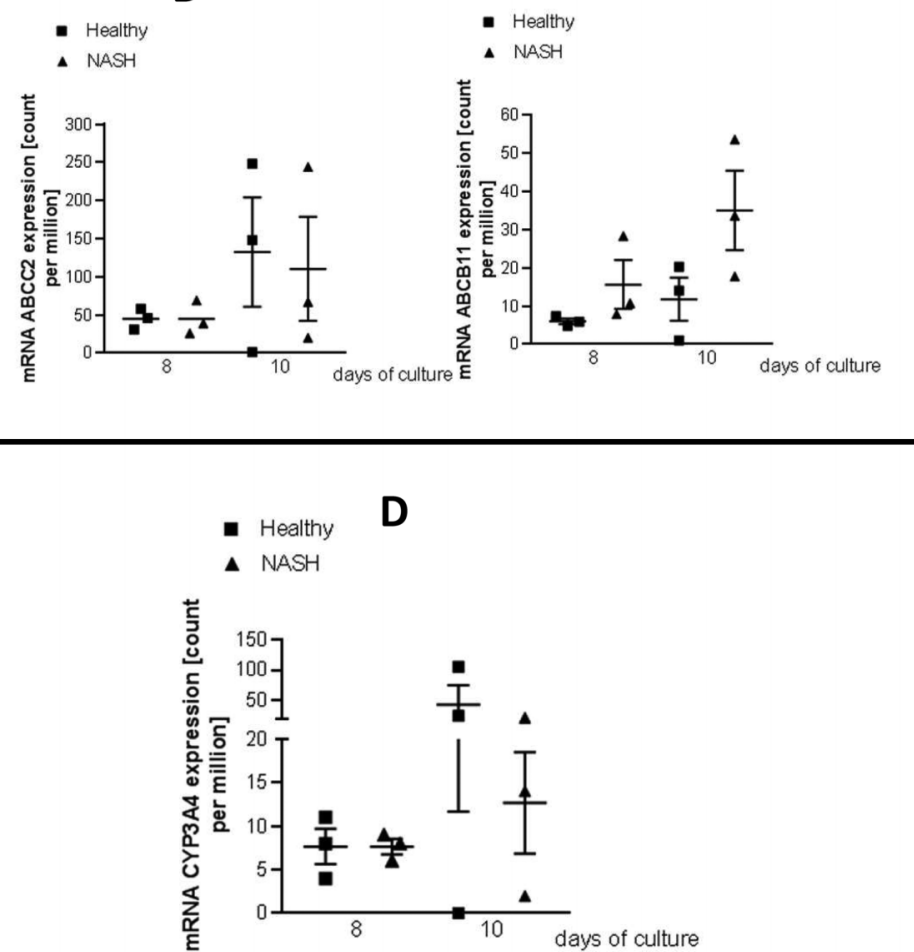

Fig. 3. PHH injury characterization in human 3D liver NASH model.

(A) Time course of adenosine triphosphate mean concentration (in RLU, +/-standard error of the mean) in 3D healthy and NASH models. (B) ABCC2 (left panel) and ABCB11 (right panel) mRNA expression in counts per million at days 8 and 10 in healthy and NASH culture. (C) Time course of CYP3A4 mean concentration (in RLU, +/-SEM) in 3D healthy and NASH models (left panel) with a focus on the fourth independent experiment trend on day 10 (right panel, each symbol represents an independent experiment). (D) CYP3A4 mRNA expression in counts per million at days 8 and 10 in healthy and NASH culture conditions. ${ }^{*} p<0.050, * * p<0.010, * * * p<0.001$, Student's test and DESeq2, respectively for panels A and C and panels B and D.

The human in vitro 3D NASH model displayed a similar stable viability as observed in the healthy $3 \mathrm{D}$ liver coculture model, whereas it showed a decreased CYP3A4 activity in three out of four experiments. Lipids droplets, characteristic of steatosis, were observed in both conditions; high basal level in healthy coculture does not allow detection of an increase of triglyceride content in NASH conditions.

\section{Human 3D liver NASH model expression of inflammatory and tissue remodeling factors}

The ability of human 3D liver coculture to react to a proinflammatory environment was explored next. The secretion of CXCL8, IL6, CXCL10 and CCL2 in supernatants of healthy and NASH 3D coculture was examined by quantifying multiplex assay at days, 6,8 and 10 . Tumor necrosis factor $-\alpha(5 \mathrm{ng} / \mathrm{mL})$ was added to the medium and changed every $2-3$ days to induce an inflammatory process.

IL6 secretion showed a significant 5-fold increase at day 6 in the 3D NASH model, as compared to the healthy condition (Fig. 5A and Supplementary Table 4), and a significant increase in gene expression was observed at days 8 and 10 (Fig. 5B). The secretion of CXCL8 was significantly up-regulated in NASH coculture at day 6 and day 8, with a 10.3-fold and 4.7-fold increase, respectively (Fig. 5A and Supplemen- tary Table 4). This observation correlated to an up-regulation in the expression of CXCL8 in 3D NASH coculture from day 8 to day 10 , with a respective fold-increase of 13.4 and 14.6 (Fig. 5B). Finally, CXCL10 secretion was also significantly upregulated in the 3D NASH model, being 32.5-fold and 22.6fold at day 6 and day 10, respectively (Fig. 5A and Supplementary Table 4). A significant 8.7-fold increase of CCL2 at day 6 was also observed in coculture supernatant in the 3D NASH model (Fig. 5C and Supplementary Table 3).

The expression of the metalloproteinases MMP2 and MMP9 involved in tissue remodeling and markers of early fibrotic events was also explored. MMP2 secretion in the human 3D NASH coculture significantly increased by 1.2 -fold, 1.7 -fold and 1.4 -fold at days 6,8 and $10 \mathrm{~m}$ respectively (Fig. 6A and Supplementary Table 4), and correlated with a significative up-regulation in MMP2 gene expression at day 10 (Fig. 6B and Supplementary Table 3). Finally, MMP9 transcripts significantly increased both at days 8 and 10, with a 3.2-fold and 6.6-fold change in the NASH environment (Fig. 6C and Supplementary Table 3).

Together, these results show that the human in vitro 3D $\mathrm{NASH}$ model is able to simulate a stable inflammatory and early fibrotic environment with the secretion of IL6, CXCL8, CXCL10, CCL2, MMP2 and MMP9 expression, respectively, as compared to the healthy model. 
Duriez M. et al: A human cellular NASH model

A

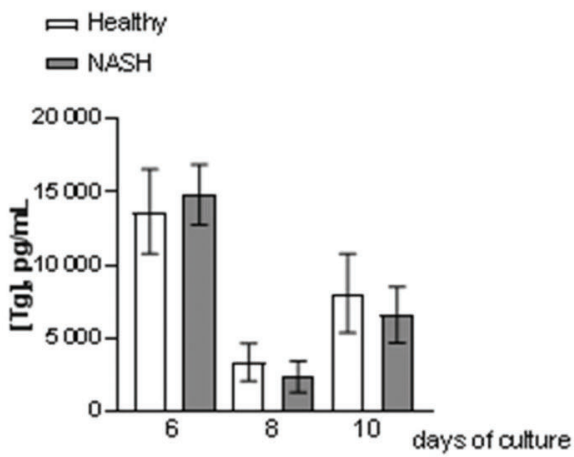

B
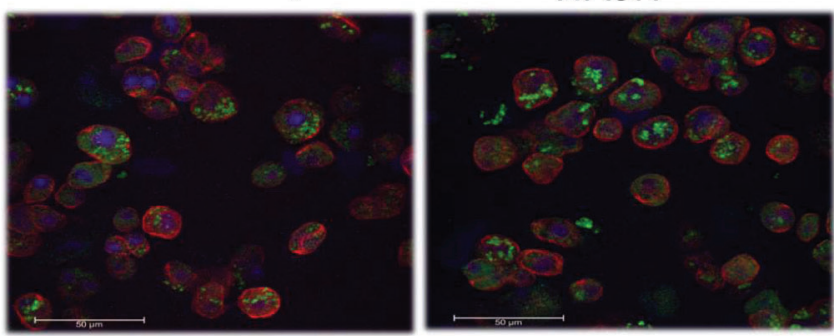

Day 8
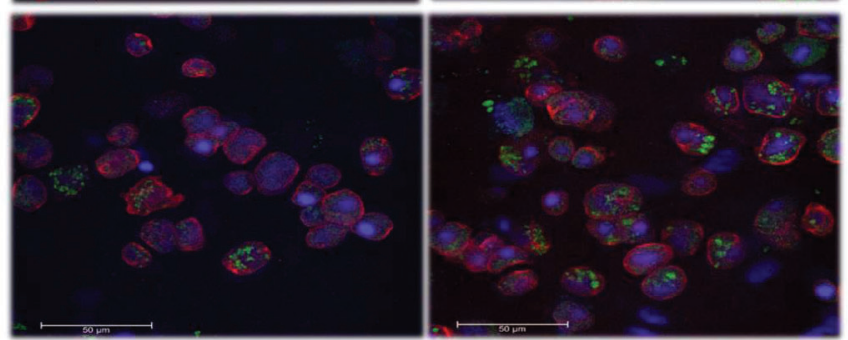

Day 10

Fig. 4. Assessment of steatosis feature.

(A) Time course of triglyceride mean concentration content (in pg/mL, +/-standard error of the mean) in 3D healthy and NASH models. (B) Lipid droplet staining in healthy and NASH culture conditions (left and right panels respectively) at days 8 and 10 (upper and lower panels, respectively). Lipid droplets are stained in green, nucleus in blue, and CK18 staining at PHH cell surface in red. Images were acquired with a Leica SP8X confocal microscope. $* p<0.050, * * p<0.010, * * * p<0.001$, Student's test.

A
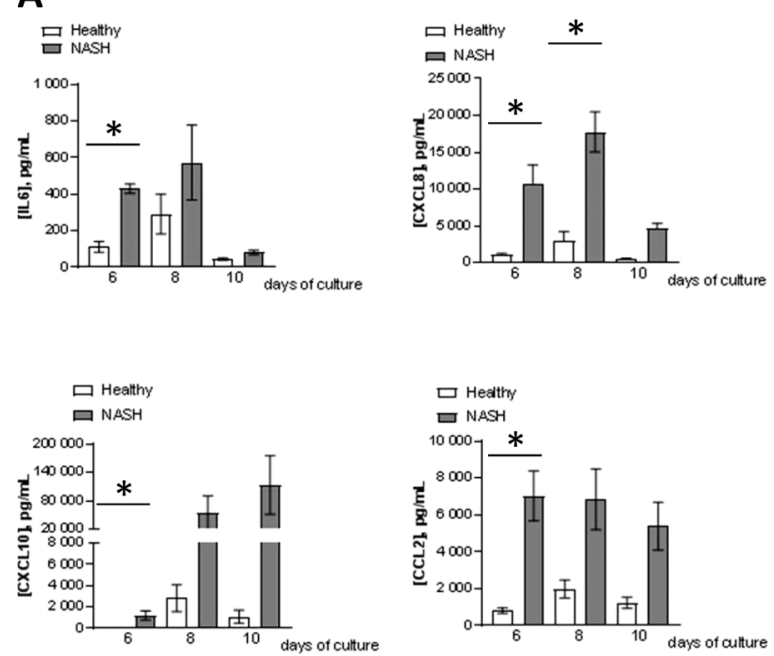

B
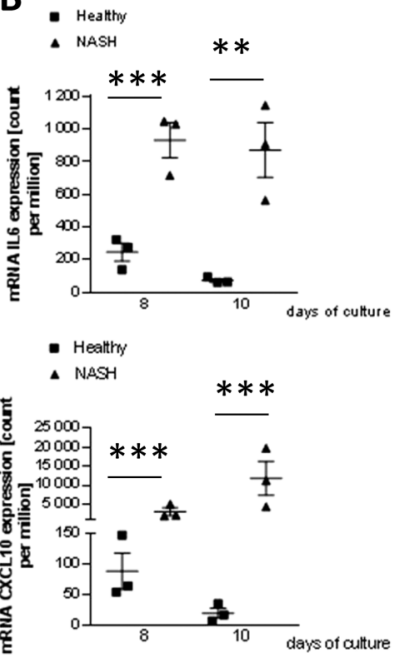

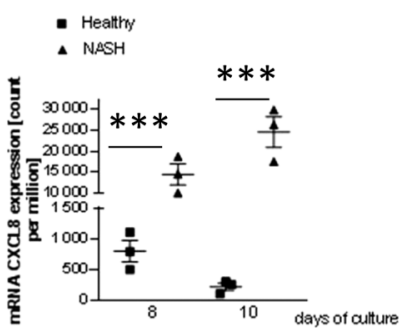

- Healthy

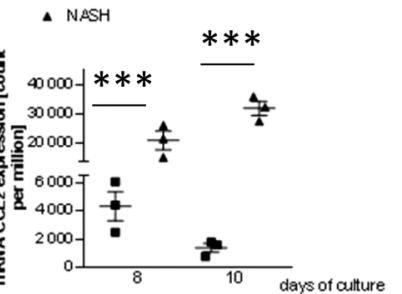

Fig. 5. Inflammatory environment induced in 3D liver NASH model.

(A) Time course of secreted IL6, CXCL8, CXCL10 and CCL2 mean concentration (in pg/mL, +/-standard error of the mean) at days 6 , 8 and 10 in healthy and NASH models. (B) IL6, CXCL8, CXCL10 and CCL2 mRNA expression in count per millions at days 8 and 10 in healthy and NASH conditions. ${ }^{*} p<0.050, * * p<0.010, * * * p<0.001$, Student's test and DESeq2, respectively, for panel A and panel B.

\section{Human 3D NASH model regulates a gene set related to pathways involved in NASH development}

To better characterize the human in vitro 3D NASH model, a global gene expression analysis has been performed by RNASeq. For this purpose, three samples from days 3,8 and 10 of the healthy $3 \mathrm{D}$ cocultures and from days 8 and 10 of the in vitro 3D NASH model were processed. Global RNA-Seq datasets from the $3 \mathrm{D}$ healthy and NASH models were first interrogated using a principal component analysis to cluster these two models during in vitro development (Fig. 7A). A distinct sample separation was observed between 3D healthy and NASH models (represented by circles and triangle, respectively, in Fig. 7A), highlighting that NASH culture condition alters the overall gene expression pattern of 3D liver cocultures. A time effect on both the healthy and NASH $3 \mathrm{D}$ liver model was also visualized, being more noticeable between days 8 and 10 in the 3D NASH coculture. 

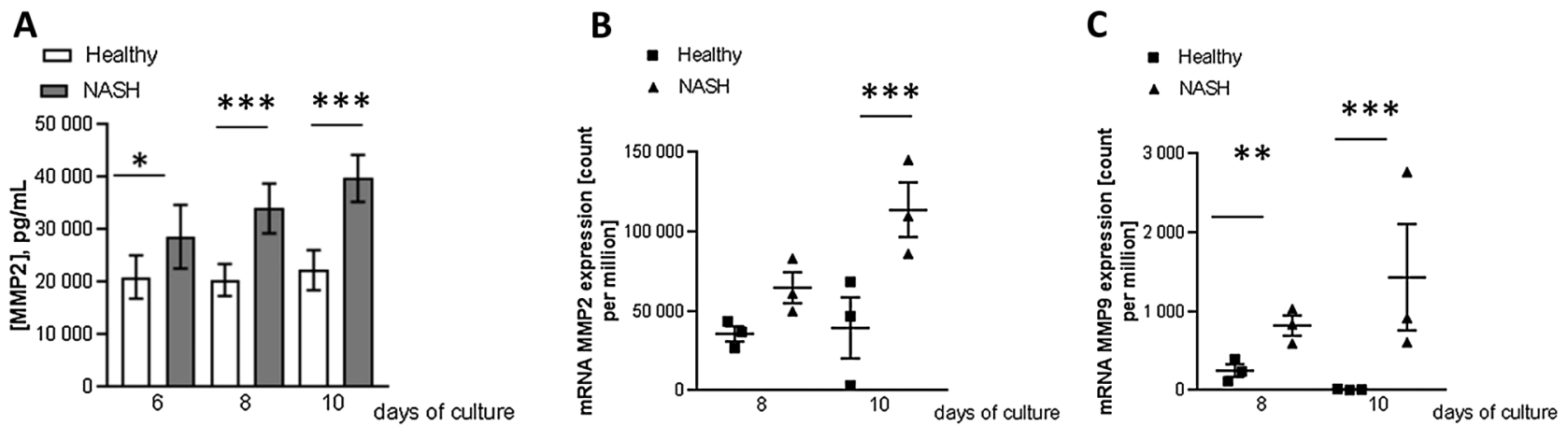

Fig. 6. Early fibrotic tissue remodeling factors induced in 3D liver NASH model

(A) Time course of secreted MMP2 mean concentration (in pg/mL, +/-standard error of the mean) at days 6,8 and 10 in healthy and NASH models. (B) MMP2 and (C) MMP9 mRNA expression level in count per millions at days 8 and 10 in healthy and NASH conditions. $* p<0.050, * * p<0.010$, $* * * p<0.001$, Student's test and DESeq2, respectively, for panel A and panels B and C.

A

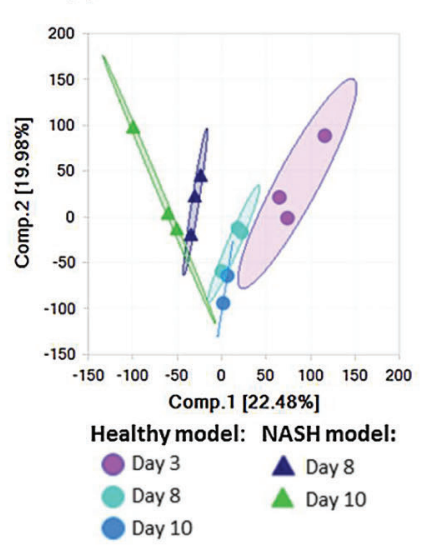

B

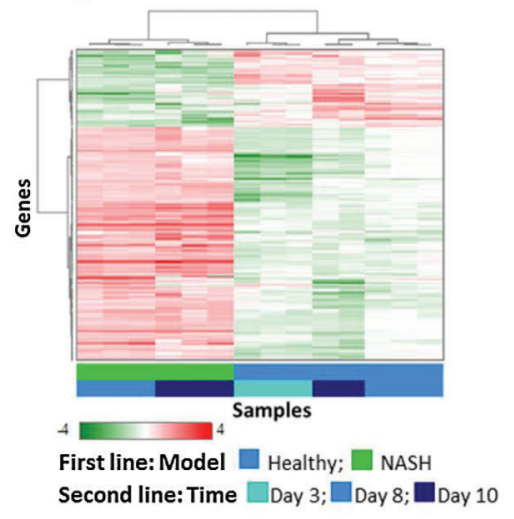

C

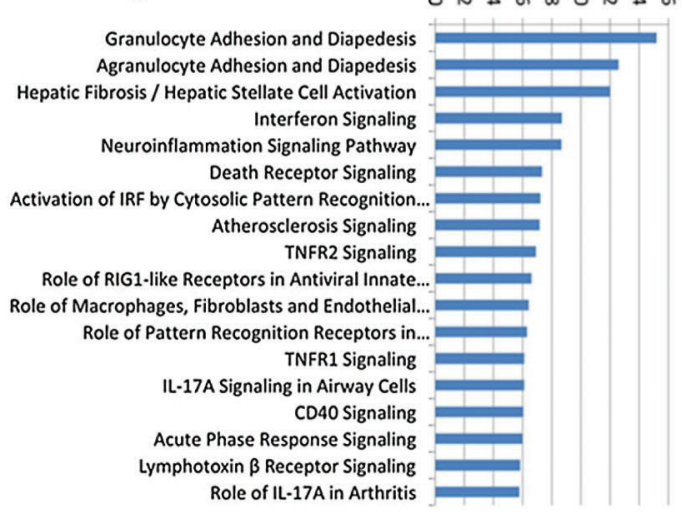

Fig. 7. RNA-Seq analysis of NASH and healthy 3D models.

(A) Principal component analysis (PCA) for gene expression in the 3D model of NASH versus healthy state at day 3,8 and 10 . The PCA was performed using DESeq2 normalized expression data. (B) Clustering analysis of differentially-expressed genes between the 3D model of the NASH disease and biological healthy models, at day 8 . Heat map illustrating unsupervised hierarchical clustering of the 468 genes specifically regulated in $\mathrm{NASH}$ model versus the biologically healthy model at day 8 (log2 of DESeq normalized data). (C) Enrichment analysis of molecular pathways in NASH model at day 8. Visualization of top 18 enriched canonical pathways in human NASH patients as compared to normal controls. Values are expressed as -log ( $p$-value).

To identify pathways modulated under $\mathrm{NASH}$-inducing conditions, differentially-expressed genes between 3D healthy and NASH models were identified. A total of 659 significant differentially-expressed genes were detected between 3D healthy and NASH conditions at day 8 (Supplementary Fig. 2). A gene subset was already modulated between day 3 and day 8 in the healthy 3D model and, thus, could not be assigned to the NASH culture condition. In the end, we selected 468 differentially-expressed genes in the 3D NASH model that were not found to be regulated over time in the healthy model. This list included 351 upregulated and 117 down-regulated genes (Supplementary Fig. 2). A hierarchical clustering analysis of healthy and NASH 3D liver models using these 468 differentially-expressed genes confirmed a good separation of the samples from healthy and NASH 3D coculture conditions (Fig. 7B). In addi- tion, most of the differentially-expressed genes after 8 days in vitro are still differentially regulated at day 10 .

We ran further pathway analysis of the 468-gene set differentially regulated in NASH conditions. Interestingly, among the top 18 enriched pathways, genes involved in hepatic fibrosis/ hepatic stellate cell activation were found to be modulated, and this pathway was ranked in third position (Fig. 7C). The first two most strongly modulated gene clusters are associated with the activation of immune cell adhesion and the inflammatory process via the diapedesis pathways (Fig. 7C).

More precisely, genes encoding for claudin adhesion proteins, together with members of the immunoglobulin superfamily, such as ICAM1, ICAM2 and VCAM1, were up-regulated (Fig. 8). Other additional up-regulated inflammation/immune pathways included interferon signaling, IRF activation, tumor necrosis factor-receptor, and IL17 signaling. 
A

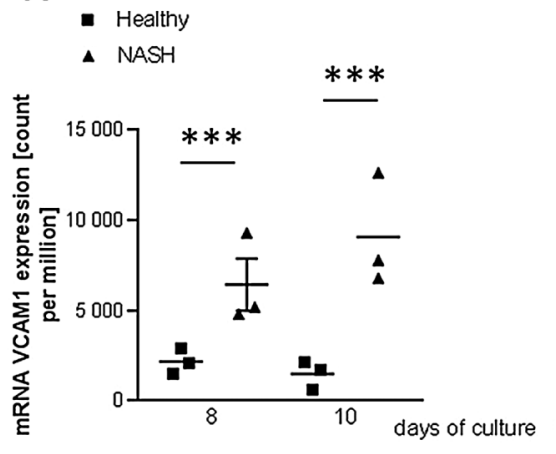

B

- Healthy

- NASH

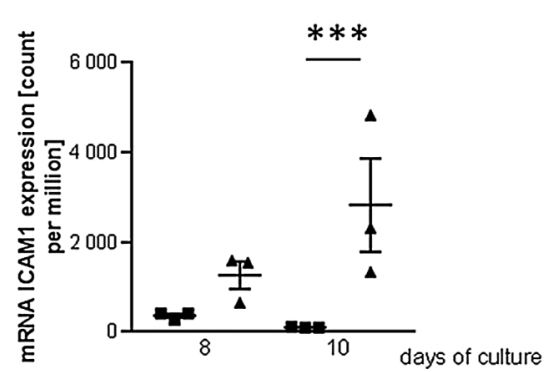

C - Healthy - $\mathrm{NASH}$

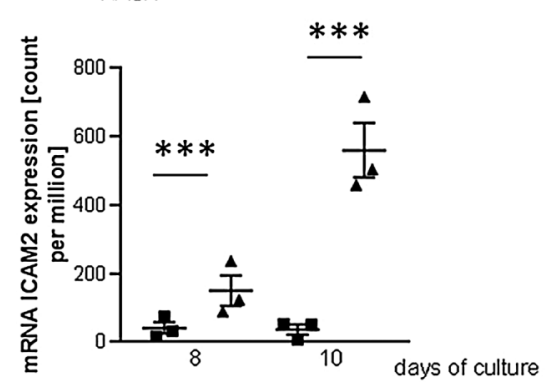

Fig. 8. mRNA expression level.

The values are expressed in counts per million at days 8 and 10 in the healthy and NASH conditions, with a scatter plot representation for VCAM1 (A), ICAM1 (B) and ICAM2 (C). * $*^{*}<0.050, * * p<0.010, * * * p<0.001$, DESeq2 test.

In conclusion, global gene expression analysis showed similar results to biochemical analysis. Together with the steatosis pathways observed under healthy conditions and maintained in the NASH 3D coculture, inflammation and early induction of fibrosis were shown to be induced in the human primary 3D liver NASH model.

\section{Discussion}

In our present study, we have set up an in vitro 3D NASH model by coculturing four human primary liver cell types, including $\mathrm{PHH}, \mathrm{HSCs}$, LECs, and KCs. The major challenge prior to this had been to develop a medium able to maintain these four primary cell types in culture, during an extended period of time, and to preserve the $\mathrm{PHH}$ mature phenotype.

Our custom Liver CoCulture media, optimized for 3D coculture, enabled us to successfully ensure cell viability up to 2 weeks in vitro. Transcriptomic analyses performed at day 10 indicated that $\mathrm{PHH}$ were still polarized and functional, with the detection of $A B C C 2, A B C B 11, C Y P 3 A 4$, and CYP7A1 gene expression.

In $\mathrm{NASH}$, injured steatotic hepatocytes induce an inflammatory environment, leading to HSC activation and fibrosis. Since this mechanism results from hepatocyte ballooning, reflecting cell death, and the 3D NASH model requires a sustained viability to perform analysis, an inflammatory stimulus was provided by adding tumor necrosis factor- $\alpha$ cytokine to the NASH culture media. PHH injury was mainly characterized by a decrease of CYP3A4 activity and mRNA expression at day $10 .^{24}$

Intriguingly, the 3D liver coculture exhibited high basal levels of triglycerides, as well as significant lipid droplet staining related to steatosis. An enrichment of pathways governing HSC activation and promoting early fibrosis was also observed. Thus, the human 3D liver coculture model displays steatosis and an activation of HSC leading to fibrosis, which are two main characteristics of NAFL/NASH. This model is able to respond to the inflammatory environment and to enhance the expression of proinflammatory cytokines, like IL6, as described in the literature. ${ }^{25,26}$ Several previous studies have associated an increased CCL2 chemokine with steatohepatitis in chronic hepatic injury through an enhancement of proinflammatory monocyte/macrophage influx in the liver. ${ }^{27-30}$ Thus, CCL2 is thought to link steatosis and inflam- mation, and accordingly, its expression is up-regulated in the human 3D NASH model.

An increase of CXCL8 and CXCL10 was also observed in the 3D liver NASH model, confirming the induction of an inflammatory environment, which is a key characteristic of NASH disease. ${ }^{31}$ As previously described, CXCL8 could be a marker of NASH combined with diabetes. ${ }^{32}$ Recently, Zimmermann et al. ${ }^{33}$ established a positive correlation between CXCL8 mRNA expression and liver fibrosis stages, showing that a high level of transcript is found in a mouse model of severe F4 fibrosis; furthermore, an up-regulation of CXCL8binding CXCR1 and CXCR2 receptors was positively correlated with chronic liver diseases. CXCL10 cytokine expression has also been correlated with fibrosis score. ${ }^{34}$ The pivotal role of CXCL10 in NASH has also been shown in vivo using CXCL10-deficient mice, since a decrease in liver steatosis, injury, inflammation and fibrosis have been reported in this model compared to wild-type animals. ${ }^{35}$

The increase of CXCL10 mRNA and secreted protein in 3D coculture maintained in the NASH-like environment, together with the key role of CXCL10 in NASH disease reported in the literature, underscores the relevance of our human 3D NASH model. ${ }^{35,36}$ Fibrogenesis is known to be associated with the synthesis and the activity of matrix metalloproteases that regulate ECM turnover during hepatic fibrosis. Among them, MMP2 and MMP9 are induced in fibrotic livers and are involved in the early disruption of the ECM in "pathologic" liver. Moreover, up-regulation of MMP2 has been identified in human fibrotic liver, whereas MMP9 induction has been highlighted in the rodent NASH model. ${ }^{37-40}$ Phenotypes that manifest later in the disease lifetime, such as collagen formation, have not been observed in this model because of the limited duration of the coculture.

The translatability of the model is further strengthened by the differential regulation of the serum endothelial dysfunction markers, ICAM-1 and VCAM-1. These two markers are of major interest to validate the induced fibrosis in the NASH model. Indeed, ICAM-1 has been found to be significantly higher in serum from NASH patients compared to serum of NAFL and healthy patients, ${ }^{41}$ supporting the role of ICAM-1 as a potential disease progression biomarker. Regarding VCAM-1, it has also been recently validated as an accurate biomarker of fibrosis in NASH patients. ${ }^{42}$ Furthermore, ASK1/MAP3K5 


\begin{tabular}{|c|c|c|c|c|c|}
\hline \multirow[b]{2}{*}{ Characteristics } & \multicolumn{5}{|c|}{ Models } \\
\hline & $\begin{array}{l}\text { Liver-on-a- } \\
\text { chip }^{49}\end{array}$ & Spheroids ${ }^{50,51}$ & $\begin{array}{l}\text { Hemoshear model } \\
\text { (Transwell) }^{15}\end{array}$ & $\begin{array}{l}\text { 3D Insight }{ }^{\mathrm{TM}} \\
\text { Human Liver } \\
\text { Microtissues } \\
\text { (spheroids) }^{48}\end{array}$ & Our 3D model \\
\hline Cell types & $\begin{array}{l}\text { HepG2 } \\
\text { cell line }\end{array}$ & $\begin{array}{l}\mathrm{PHH} \text { and small } \\
\text { amounts of KCs } \\
\text { and HSCs }\end{array}$ & $\mathrm{PHH}, \mathrm{M \Phi}$ and $\mathrm{HSCs}$ & $\begin{array}{l}\mathrm{PHH}, \mathrm{KCs}, \mathrm{HSCs} \\
\text { and LECs }\end{array}$ & $\begin{array}{l}\mathrm{PHH}, \mathrm{KCs}, \mathrm{HSCs} \\
\text { and LECs }\end{array}$ \\
\hline Stability time & 8 days & 35 days & 10 days & 9 days & 10 days \\
\hline Hepatocyte integrity & $\checkmark$ & $\checkmark$ & $\checkmark$ & $\checkmark$ & $\checkmark$ \\
\hline Steatosis induction & $\checkmark$ & $\checkmark$ & $\checkmark$ & $\checkmark$ & $\checkmark$ \\
\hline Inflammation induction & $x$ & IL6 secretion only & $\checkmark$ & $\checkmark$ & $\checkmark$ \\
\hline Fibrosis induction & $x$ & $x$ & $\checkmark$ & $\checkmark$ & $\checkmark$ \\
\hline Disease induction & NAFL & NAFL & NASH & NASH & NASH \\
\hline NASH markers & $x$ & $x$ & $x$ & $x$ & $\checkmark$ \\
\hline
\end{tabular}

has been largely described in the literature as a pharmacological target for NASH disease treatment ${ }^{43-45}$ and, interestingly, its up-regulation is observed in our human 3D NASH model (Supplementary Table 2).

Finally, PD-L1 (CD274) gene expression was shown to be enhanced in the human 3D NASH model, and this finding is relevant for the induction of fibrosis. An up-regulation of the PD-L1 gene has been described previously in injured liver and associated with HSC immunomodulatory activity ${ }^{46}$ and hepatocyte damage leading to inflammatory processes. ${ }^{47}$ The induction of PD-L1 and PDCD1LG2 in the human 3D NASH model suggests that this pathway could qualify as an attractive avenue for NASH treatment.

Several 3D models for NASH have been developed and a recent comprehensive comparison was reported by Oseini et al. ${ }^{14}$ Most of these models are used to measure drug toxicity and clearance but do not mimic disease progression. Few 3D culture systems have been set up for NAFLD disease modeling. The main features are summarized in Table 1. For example, liver-on-a-chip and spheroid technology have been used with cell lines or primary cell types to induce steatosis and recapitulate NAFLD. Two different commercial technologies, the Transwell ${ }^{\mathrm{TM}}$ from Hemoshear device and 3D Insight ${ }^{\mathrm{TM}}$ Human Liver Microtissues from InSphero (Table 1 , rows $3 \& 4$ ), are comparable to our model in terms of model viability and NASH features. However, in the Transwell model, it is necessary to differentiate monocytes in macrophages. Furthermore, the use of a microfluidic system hampers its application for screening purposes. More recently, Mukherjee et al. ${ }^{48}$ has developed a 3D model using 3D Insight Human liver microtissues that is the closest to the one reported in this paper. With the four human primary cell types, they described NASH features and use reference molecules to reverse the disease. However, transcriptional characterization was not reported. In our model, the translatability, as typified by NASH transcriptional markers, is more thoroughly defined. Our model is also distinguished by the fact that embedded endothelial cells were used. These cells have been shown to play a pivotal role in NAFLD/NASH progression from the simple steatosis to the early NASH stage probably by activating HSCs and KCs. ${ }^{16,18}$ It has been suggested that coculture of primary human HSCs and LECs from cirrhotic livers promote fibrillar collagen production by HSCs. In addition, it is thought that liver sinusoidal endothelial cells injuries in the early stage of NAFLD are necessary for the activation of KCs and HSCs, and therefore for the NAFLD/NASH progression.

In conclusion, we have developed a new 3D NASH model with four human primary cell types, which include liver sinusoidal endothelial cells.

Optimization of the model, by using microfluidic devices, could be an option to increase cell survival and to mimic chronic disease, but it will likely not be suitable for screening purposes. Our 3D NASH model could be maintained for 10 days in vitro and showed triglyceride content leading to steatosis, an inflammatory response and activation of fibrosisrelated pathways that are also associated with NASH. The relative extended life time of the $3 \mathrm{D}$ model culture makes it an attractive platform to evaluate preventive and curative treatments with drug candidates. These experiments will be reported in due course.

\section{Acknowledgments}

The authors want to thank Prof. François Pattou and Prof. Bart Staels for support and advice, and the Federation Hospital \& University INTEGRA, who in association with SANOFI are part of the PreciNASH consortium.

\section{Funding}

This work was supported by grants from the Agence Nationale pour la Recherche (ANR-16-RHUS-0006-PreciNASH).

\section{Conflict of interest}

All of the authors except TS were employees of Sanofi during the course of the study, and all are or have been shareholders of Sanofi. 


\section{Author contributions}

Study design, data acquisition, and biochemical data analysis $(\mathrm{AJ}, \mathrm{LH})$, transcriptional data acquisition and analysis (SR, $M D B, C R)$, imaging data $(\mathrm{GH})$, critical revision of the manuscript (XV and ZB), statistical analysis (TS, VM), analyzing and interpreting data, critical revision of the manuscript, and funding acquisition (JCG, MD, AK, CO, VM), study supervision, study design, data acquisition analysis and interpretation, and drafting the manuscript (MD, ACLF).

\section{References}

[1] Younossi Z, Tacke F, Arrese M, Chander Sharma B, Mostafa I, Bugianesi E, et al. Global perspectives on nonalcoholic fatty liver disease and nonalcoholic steatohepatitis. Hepatology 2019;69:2672-2682. doi: 10.1002/hep.30251.

[2] Friedman SL, Neuschwander-Tetri BA, Rinella M, Sanyal AJ. Mechanisms of NAFLD development and therapeutic strategies. Nat Med 2018;24:908-922. doi: 10.1038/s41591-018-0104-9.

[3] Buzzetti E, Pinzani M, Tsochatzis EA. The multiple-hit pathogenesis of nonalcoholic fatty liver disease (NAFLD). Metabolism 2016;65:1038-1048. doi: 10.1016/j.metabol.2015.12.012.

[4] Younossi ZM, Koenig AB, Abdelatif D, Fazel Y, Henry L, Wymer M. Global epidemiology of nonalcoholic fatty liver disease-Meta-analytic assessment of prevalence, incidence, and outcomes. Hepatology 2016;64:73-84. doi: 10.1002/hep. 28431.

[5] Dulai PS, Singh S, Patel J, Soni M, Prokop L], Younossi Z, et al. Increased risk of mortality by fibrosis stage in nonalcoholic fatty liver disease: Systematic review and meta-analysis. Hepatology 2017;65:1557-1565. doi: 10.1002/hep.29085.

[6] Lassailly G, Caiazzo R, Buob D, Pigeyre M, Verkindt $H$, Labreuche J, et al. Bariatric surgery reduces features of nonalcoholic steatohepatitis in morbidly obese patients. Gastroenterology 2015;149:379-388; quiz e15-e16. doi: 10.1053/j.gastro.2015.04.014.

[7] Vilar-Gomez E, Martinez-Perez Y, Calzadilla-Bertot L, Torres-Gonzalez A, GraOramas B, Gonzalez-Fabian $L$, et al. Weight loss through lifestyle modification significantly reduces features of nonalcoholic steatohepatitis. Gastroenterology 2015;149:367-378.e5; quiz e14-e15. doi: 10.1053/j.gastro.2015.04.005.

[8] Rinella ME, Sanyal AJ. Management of NAFLD: a stage-based approach. Nat Rev Gastroenterol Hepatol 2016;13:196-205. doi: 10.1038/nrgastro.2016.3.

[9] Santhekadur PK, Kumar DP, Sanyal AJ. Preclinical models of non-alcoholic fatty liver disease. J Hepatol 2018;68:230-237. doi: 10.1016/j.jhep.2017. 10.031 .

[10] Godoy P, Hewitt NJ, Albrecht U, Andersen ME, Ansari N, Bhattacharya S, et al. Recent advances in 2D and $3 \mathrm{D}$ in vitro systems using primary hepatocytes, alternative hepatocyte sources and non-parenchymal liver cells and their use in investigating mechanisms of hepatotoxicity, cell signaling and ADME. Arch Toxicol 2013;87:1315-1530. doi: 10.1007/s00204-013-1078-5.

[11] Martinez-Hernandez A, Amenta PS. The hepatic extracellular matrix. II. Ontogenesis, regeneration and cirrhosis. Virchows Arch A Pathol Anat Histopathol 1993;423:77-84. doi: 10.1007/BF01606580.

[12] Wells RG. Cellular sources of extracellular matrix in hepatic fibrosis. Clin Liver Dis 2008;12:759-768. doi: 10.1016/j.cld.2008.07.008.

[13] Abedin M, King N. Diverse evolutionary paths to cell adhesion. Trends Cell Biol 2010;20:734-742. doi: 10.1016/j.tcb.2010.08.002

[14] Oseini AM, Cole BK, Issa D, Feaver RE, Sanyal AJ. Translating scientific discovery: the need for preclinical models of nonalcoholic steatohepatitis. Hepatol Int 2018;12:6-16. doi: 10.1007/s12072-017-9838-6.

[15] Feaver RE, Cole BK, Lawson MJ, Hoang SA, Marukian S, Blackman BR, et al. Development of an in vitro human liver system for interrogating nonalcoholic steatohepatitis. JCI Insight 2016;1:e90954. doi: 10.1172/jci.insight. 90954

[16] Ramachandran P, Dobie R, Wilson-Kanamori JR, Dora EF, Henderson BEP, LuU NT, et al. Resolving the fibrotic niche of human liver cirrhosis at single-cell level. Nature 2019;575:512-518. doi: 10.1038/s41586-019-1631-3.

[17] Alkhouri N, Dixon LJ, Feldstein AE. Lipotoxicity in nonalcoholic fatty liver disease: not all lipids are created equal. Expert Rev Gastroenterol Hepatol 2009;3:445-451. doi: 10.1586/egh.09.32.

[18] Miyao M, Kotani H, Ishida T, Kawai C, Manabe S, Abiru H, et al. Pivotal role of liver sinusoidal endothelial cells in NAFLD/NASH progression. Lab Invest 2015;95:1130-1144. doi: 10.1038/labinvest.2015.95.

[19] Hu J, Ge H, Newman M, Liu K. OSA: a fast and accurate alignment tool for RNASeq. Bioinformatics 2012;28:1933-1934. doi: 10.1093/bioinformatics/bts294.

[20] Love MI, Huber W, Anders S. Moderated estimation of fold change and dispersion for RNA-seq data with DESeq2. Genome Biol 2014;15:550. doi: 10. 1186/s13059-014-0550-8.

[21] Benjamini Y, Hochberg Y. Controlling the false discovery rate: A practical and powerful approach to multiple testing. Journal of the Royal Statistical Society
Series B (Methodological) 1995;57:289-300. doi: 10.1111/j.2517-6161. 1995.tb02031.x

[22] Krämer A, Green J, Pollard J Jr, Tugendreich S. Causal analysis approaches in Ingenuity Pathway Analysis. Bioinformatics 2014;30:523-530. doi: 10. 1093/bioinformatics/btt703.

[23] Kolios G, Valatas V, Kouroumalis E. Role of Kupffer cells in the pathogenesis of liver disease. World J Gastroenterol 2006;12:7413-7420. doi: 10. 3748/wjg.v12.i46.7413.

[24] Woolsey S], Mansell SE, Kim RB, Tirona RG, Beaton MD. CYP3A activity and expression in nonalcoholic fatty liver disease. Drug Metab Dispos 2015;43: 1484-1490. doi: 10.1124/dmd.115.065979.

[25] Bocsan IC, Milaciu MV, Pop RM, Vesa SC, Ciumarnean L, Matei DM, et al. Cytokines genotype-phenotype correlation in nonalcoholic steatohepatitis. Oxid Med Cell Longev 2017;2017:4297206. doi: 10.1155/2017/4297206.

[26] Rabelo F, Oliveira CP, Faintuch J, Mazo DF, Lima VM, Stefano JT, et al. Pro- and anti-inflammatory cytokines in steatosis and steatohepatitis. Obes Surg 2010;20:906-912. doi: 10.1007/s11695-010-0181-4.

[27] Vucur M, Gassler N, Huss S, Klussmann S, Eulberg D, Luedde T, et al. Pharmacological inhibition of the chemokine CCL2 (MCP-1) diminishes liver macrophage infiltration and steatohepatitis in chronic hepatic injury. Gut 2012; 61:416-426. doi: 10.1136/gutjinl-2011-300304.

[28] Tosello-Trampont AC, Landes SG, Nguyen V, Novobrantseva TI, Hahn YS. Kuppfer cells trigger nonalcoholic steatohepatitis development in dietinduced mouse model through tumor necrosis factor- $\alpha$ production. J Biol Chem 2012;287:40161-40172. doi: 10.1074/jbc.M112.417014.

[29] Narayanan S, Surette FA, Hahn YS. The immune landscape in nonalcoholic steatohepatitis. Immune Netw 2016;16:147-158. doi: 10.4110/in.2016.16.3.147.

[30] Krenkel O, Tacke F. Liver macrophages in tissue homeostasis and disease. Nat Rev Immunol 2017;17:306-321. doi: 10.1038/nri.2017.11.

[31] Koyama Y, Brenner DA. Liver inflammation and fibrosis. ] Clin Invest 2017; 127:55-64. doi: 10.1172/JCI88881.

[32] Estep JM, Baranova A, Hossain N, Elariny H, Ankrah K, Afendy A, et al. Expression of cytokine signaling genes in morbidly obese patients with non-alcoholic steatohepatitis and hepatic fibrosis. Obes Surg 2009;19: 617-624. doi: 10.1007/s11695-009-9814-x.

[33] Zimmermann HW, Seidler S, Gassler N, Nattermann J, Luedde T, Trautwein C, et al. Interleukin-8 is activated in patients with chronic liver diseases and associated with hepatic macrophage accumulation in human liver fibrosis. PLoS One 2011;6:e21381. doi: 10.1371/journal.pone.0021381.

[34] Domagalski K, Pawłowska M, Kozielewicz D, Dybowska D, Tretyn A, Halota W. The impact of IL28B genotype and liver fibrosis on the hepatic expression of IP10, IFI27, ISG15, and MX1 and their association with treatment outcomes in patients with chronic hepatitis C. PLoS One 2015;10:e0130899. doi: 10. 1371/journal.pone.0130899.

[35] Zhang X, Shen J, Man K, Chu ES, Yau TO, Sung JC, et al. CXCL10 plays a key role as an inflammatory mediator and a non-invasive biomarker of non-alcoholic steatohepatitis. J Hepatol 2014;61:1365-1375. doi: 10.1016/j.jhep. 2014.07.006.

[36] Tomita K, Freeman BL, Bronk SF, LeBrasseur NK, White TA, Hirsova P, et al. CXCL10-mediates macrophage, but not other innate immune cells-associated inflammation in murine nonalcoholic steatohepatitis. Sci Rep 2016;6: 28786. doi: $10.1038 /$ srep28786.

[37] Okazaki I, Noro T, Tsutsui N, Yamanouchi E, Kuroda H, Nakano M, et al. Fibrogenesis and carcinogenesis in nonalcoholic steatohepatitis (NASH): Involvement of matrix metalloproteinases (MMPs) and tissue inhibitors of metalloproteinase (TIMPs). Cancers (Basel) 2014;6:1220-1255. doi: 10. 3390/cancers6031220.

[38] Giannandrea M, Parks WC. Diverse functions of matrix metalloproteinases during fibrosis. Dis Model Mech 2014;7:193-203. doi: 10.1242/dmm. 012062.

[39] Robert S, Gicquel T, Victoni T, Valença S, Barreto E, Bailly-Maître B, et al. Involvement of matrix metalloproteinases (MMPs) and inflammasome pathway in molecular mechanisms of fibrosis. Biosci Rep 2016;36:e00360. doi: 10.1042/BSR20160107.

[40] Friedman SL. Liver fibrosis - from bench to bedside. J Hepatol 2003;38 Suppl 1:S38-S53. doi: 10.1016/s0168-8278(02)00429-4.

[41] Ito S, Yukawa T, Uetake S, Yamauchi M. Serum intercellular adhesion molecule1 in patients with nonalcoholic steatohepatitis: comparison with alcoholic hepatitis. Alcohol Clin Exp Res 2007;31:S83-S87. doi: 10.1111/j.1530-0277. 2006.00292.x.

[42] Lefere S, Van de Velde F, Devisscher L, Bekaert M, Raevens S, Verhelst X, et al. Serum vascular cell adhesion molecule-1 predicts significant liver fibrosis in non-alcoholic fatty liver disease. Int J Obes (Lond) 2017;41:1207-1213. doi: $10.1038 /$ ijo.2017.102.

[43] Povsic M, Oliver L, Jiandani NR, Perry R, Bottomley J. A structured literature review of interventions used in the management of nonalcoholic steatohepatitis (NASH). Pharmacol Res Perspect 2019;7:e00485. doi: 10.1002/prp2. 485. 
[44] Connolly J], Ooka K, Lim JK. Future pharmacotherapy for non-alcoholic steatohepatitis (NASH): Review of phase 2 and 3 trials. J Clin Transl Hepatol 2018;6:264-275. doi: 10.14218/JCTH.2017.00056.

[45] Drescher HK, Weiskirchen S, Weiskirchen R. Current status in testing for nonalcoholic fatty liver disease (NAFLD) and nonalcoholic steatohepatitis (NASH). Cells 2019;8:845. doi: 10.3390/cells8080845.

[46] Yu MC, Chen CH, Liang X, Wang L, Gandhi CR, Fung JJ, et al. Inhibition of Tcell responses by hepatic stellate cells via $\mathrm{B} 7-\mathrm{H} 1$-mediated T-cell apoptosis in mice. Hepatology 2004;40:1312-1321. doi: 10.1002/hep.20488.

[47] Wu JF, Hsu HY, Ni YH, Chen HL, Wu TC, Chang MH. Suppression of furin by interferon- $\gamma$ and the impact on hepatitis B virus antigen biosynthesis in human hepatocytes. Am J Pathol 2012;181:19-25. doi: 10.1016/j.ajpath. 2012.03.036.
[48] Mukherjee S, Zhelnin L, Sanfiz A, Pan J, Li Z, Yarde M, et al. Development and validation of an in vitro 3D model of NASH with severe fibrotic phenotype. Am J Transl Res 2019;11:1531-1540.

[49] Gori M, Simonelli MC, Giannitelli SM, Businaro L, Trombetta M, Rainer A. Investigating nonalcoholic fatty liver disease in a liver-on-a-chip microfluidic device. PLoS One 2016;11:e0159729. doi: 10.1371/journal.pone.0159729.

[50] Bell CC, Hendriks DF, Moro SM, Ellis E, Walsh J, Renblom A, et al. Characterization of primary human hepatocyte spheroids as a model system for druginduced liver injury, liver function and disease. Sci Rep 2016;6:25187. doi: $10.1038 /$ srep25187.

[51] Kozyra M, Johansson I, Nordling $\AA$, Ullah S, Lauschke VM, Ingelman-Sundberg $M$. Human hepatic 3D spheroids as a model for steatosis and insulin resistance. Sci Rep 2018;8:14297. doi: 10.1038/s41598-018-32722-6. 